\title{
Improving cell adhesion: development of a biosensor for cell behaviour monitoring by surface grafting of sulfonic groups onto a thermoplastic polyurethane
}

\author{
P. Alves $\cdot$ S. Pinto $\cdot$ P. Ferreira $\cdot$ Jean-Pierre Kaiser $\cdot$ \\ Arie Bruinink • Hermínio C. de Sousa • \\ M. H. Gil
}

Received: 17 May 2013/Accepted: 5 May 2014/Published online: 23 May 2014

(C) Springer Science+Business Media New York 2014

\begin{abstract}
The surface properties of a material in combination with the mechanical properties are responsible for the material performance in a biological environment as well as the behaviour of the cells which contact with the material. Surface properties such as chemical, physical, biological play an important role in the biomaterials filed. In this work, the surface of a thermoplastic polyurethane film (Elastollan ${ }^{\circledR} 1180$ A50) was tailored with sulfonic groups by grafting [2-(methacryloxyl)ethyl]-dimethyl-(3sulfopropyl)-ammonium hydroxide (SB) after a previous surface activation either by Argon plasma or by ultra-violet irradiation. This surface modification had the purpose of improving cell adhesion in order to develop a biosensor able to monitor cell behaviour. The surfaces were characterized by X-ray photoelectron spectroscopy, by atomic force microscopy and by contact angle measurements in order to evaluate the efficiency of the modification. Additionally, blood compatibility studies and cell adhesion tests with human bone marrow cells were performed. These methods allowed the grafting of SB and the results indicate that a higher density of grafting was achieved with previous surface plasma treatment than with UV irradiation. However, for both techniques, the presence of SB functional groups led to a decrease of hydrophobicity and
\end{abstract}

P. Alves $(\varangle) \cdot$ S. Pinto · P. Ferreira · H. C. de Sousa ·

M. H. Gil

CIEPQPF, Departamento de Engenharia Química, Universidade de Coimbra, Polo II, Pinhal de Marrocos, 3030-790 Coimbra,

Portugal

e-mail: palves@eq.uc.pt

J.-P. Kaiser · A. Bruinink

EMPA St. Gallen, Lerchenfeldstrasse 5, 9014 St. Gallen, CH,

Switzerland roughness of the surface, together with an improvement of the materials biological performance.

\section{Introduction}

The use of commercial polymers in the most various areas has been increasing in the last years. Therefore, the surface of a material plays an important role since it is the surface that mediates the interaction between the material and the environment. It was in the early twentieth century that the study of interactions of surfaces began [1]. Since then, surface science has been the focus of many investigations, becoming gradually important in the field of biomaterials.

Several methods, such as chemical, physical and biological have been reported with the aim of surface modification (without affecting the bulk mechanical properties) [2]. Being the preservation of the bulk mechanical properties a crucial factor in surface modification, this work was focused on the use of radiation techniques to graft a polymeric material with a very thin monomer layer [3]. This thin layer would provide the appropriate answer in some biological environments.

The use of radiation for graft polymerization is a very clean and simple method. Commonly, radicals (reactive sites) are formed on the surface of the material. Then, these reactive sites will allow the subsequent covalent linkage of a monomer or polymer to the material [4]. This modification in the surface, either by using different gases in plasma or by ultraviolet (UV) irradiation, induces changes in wettability, adhesion, adsorption, printability, chemical reactivity and sensitivity to light.

Plasma treatment is a wide process and has been more explored in the last years and is considered to be environmental efficient due to its high efficiency associated 
with a low waste production. Plasma treatment allows the cleaning of a surface, etching, crosslinking, grafting or even the addition, substitution or formation of functional groups. The type of reaction is controlled by the active species present in the plasma [5], which are dependent on the working gas (e.g. Argon, Oxygen, Nitrogen), time of reaction, chamber type and power of the plasma process $[6$, 7].

UV light is normally combined with a photosensitizer or photoinitiator to generate free radicals within the surface of a material and consequently to achieve a graft polymerization on the surface of a material. Consequently, irradiation time and the depth of irradiation penetration on the grafted polymer control the extension of the grafting reaction. The concentration of photosensitizer can influence the amount of the grafted material along and within the surface [8-10].

Due to these radiation methods properties and ability to create functional surfaces, UV and plasma graft polymerization are highly attractive methods for surface modification of materials, particularly when the surfaces have no chemically reactive groups $[8,11]$. Furthermore, within the field of surface modification, these methods became extremely attractive since UV and plasma allow to modify only a thin surface layer while preserving the bulk chemical and mechanical properties of the material $[4,12]$.

The aim of the present study was to develop a material with suitable surface properties to be used in the production of a biosensor able to monitor cell behaviour by detection of traction forces at their focal adhesion points. These are connected to their cytoskeleton and work as attaching points between the cell and the material surface [13]. Since cell movement is an active process, slight effects on cell function may directly be reflected by the size of the contractile forces submitted to the underlying substratum. These forces may directly be dependent on the functional state of the cell [14]. The extent and change in contractile force is assumed to be influenced at a very early phase after test compound treatment and long before other indices of the cell state like cell shape or mitochondrial activity are changed [15, 16]. Therefore, the obtained biosensor may find its application in various fields of health care from characterising patient's specific cells and allowing enhanced treatments, to the detection of bioactive medium components having pharmacological or toxic effect on cells.

In order to obtain a suitable cell-adhering material, two different strategies were used to modify the surface of a thermoplastic polyurethane (TPU)—Elastollan ${ }^{\circledR} 1180$ A50 - to improve its hydrophilicity and promote cell adhesion without compromising its bulk properties. For this propose, Elastollan ${ }^{\circledR} 1180$ A50 was grafted with [2(methacryloxyl)ethyl]-dimethyl-3-sulfopropyl)-ammonium hydroxide (SB) by using low pressure plasma and UV irradiation. SB was selected for this purpose since other authors [17] showed that sulfobetaine monomers can improve blood compatibility and reduce platelet adhesion.

The surface properties of the modified TPU were assessed by X-ray photoelectron spectroscopy (XPS), atomic force microscopy (AFM) and contact angle measurements. Blood compatibility tests and cell adhesion behaviour with human bone marrow cells (HBMC) were also performed.

\section{Experimental}

\subsection{Materials}

[2-(methacryloxyl)ethyl]-dimethyl-3-sulfopropyl)-ammonium hydroxide $(\mathrm{SB})$ was purchased from Sigma-Aldrich and Elastollan ${ }^{\circledR} 1180 \mathrm{~A} 50$ was obtained from BASF. Isopropyl alcohol and dimethylformamide (DMF) were obtained from JMGS, Portugal. Irgacure ${ }^{\circledR} 2959$ was kindly given by CIBA (Ciba Specialty Chemicals, Basel, Switzerland). Rabbit blood with acid-citrate-dextrose (ACD) was purchased from Probiológica, Portugal. Carbonate-free HEPES buffered (25 mM) and Dulbecco's modified Eagle medium (DMEM) with $10 \%$ foetal calf serum were purchased from Life Technologies, Basel, Switzerland. All of the chemicals were used as received.

\subsection{Methods}

\subsubsection{Films preparation}

Elastollan ${ }^{\circledR} 1180$ A50 films were prepared by solvent evaporation. Elastollan ${ }^{\circledR} 1180$ A50 was solubilized in DMF to a $10 \%(\mathrm{w} / \mathrm{v})$ TPU solution. This solution was poured into glass Petri plates. Then, the Petri dishes were stored in an oven, at $60{ }^{\circ} \mathrm{C}$ for $24 \mathrm{~h}$. Afterwards, the films were removed from the dishes and ultrasonically cleaned with isopropyl alcohol for $15 \mathrm{~min}$, prior to surface grafting experiments.

\subsubsection{Plasma grafting}

A laboratory and small-scale production plasma system FEMTO (low pressure plasma), manufactured by Diener Electronics, with a stainless steel plasma chamber of $100 \mathrm{~mm}$ diameter and $270 \mathrm{~mm}$ length, was used for the plasma surface modification experiments. The TPU membranes were placed $80 \mathrm{~mm}$ from the electrode and were plasma treated with Argon for 3 min, with a chamber pressure of 0.6 mbar and 100 Watt power of plasma [6]. Afterwards, the plasma-treated TPU membrane was dipped in a $10 \%(\mathrm{v} / \mathrm{v})$ aqueous solution of SB and placed in an oven at 


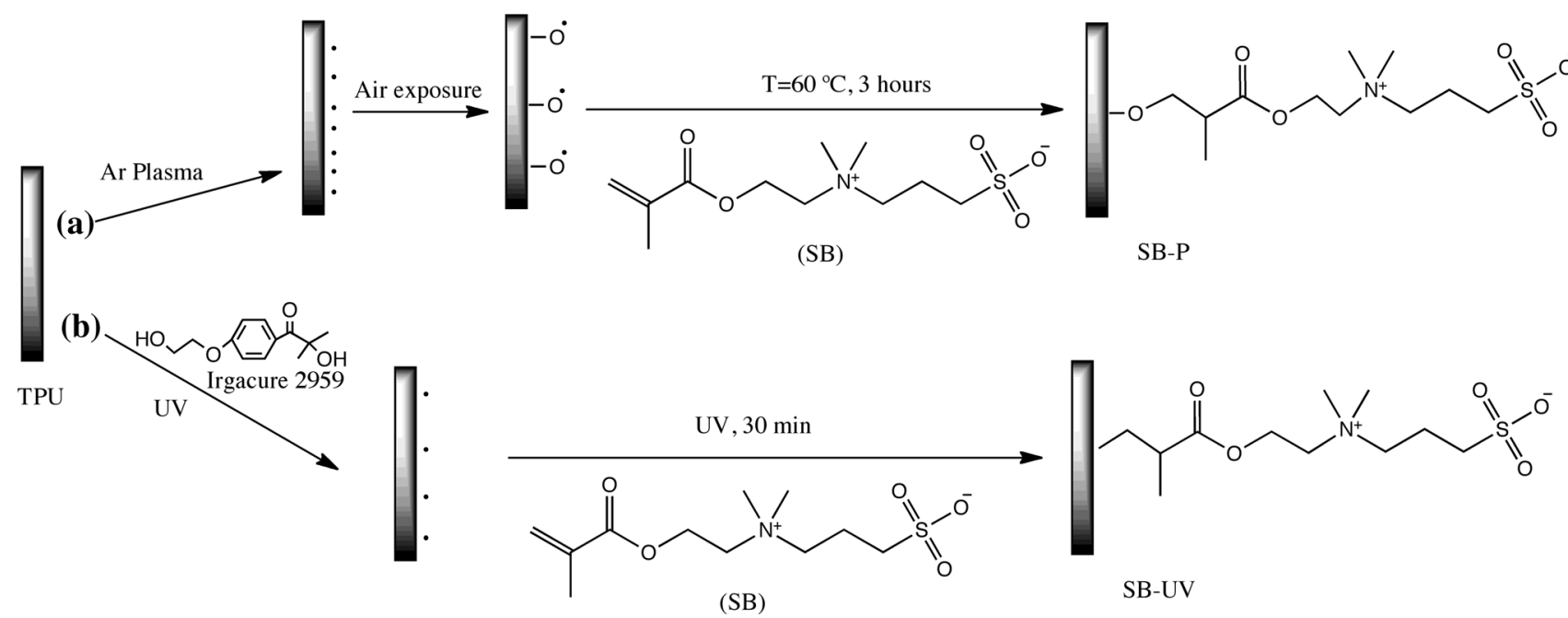

Fig. 1 Schematic representation of a Argon plasma activation followed by the SB monomer grafting and $\mathbf{b}$ UV grafting mechanism

$60{ }^{\circ} \mathrm{C}$ for $8 \mathrm{~h}$. Finally, SB-grafted films (SB-P) were abundantly washed with water and dried until constant weight. This procedure is schematically represented in Fig. 1a.

\subsubsection{UV grafting}

For the UV grafting a Mineralight ${ }^{\circledR}$ Lamp, Model UVGL48 , in the $254 \mathrm{~nm}$ wavelength setting with a power of 6 Watt was used. The samples were placed at a distance of $4 \mathrm{~cm}$ from the light source. The films were first activated with UV light in a $0.5 \%$ photoinitiator (Irgacure ${ }^{\circledR} 2959$ ) aqueous solution for $30 \mathrm{~min}$. Then, the membranes were removed from the Irgacure ${ }^{\circledR} 2959$ solution and dipped into a $10 \%(\mathrm{v} / \mathrm{v}) \mathrm{SB}$ aqueous solution and irradiated a second time with UV light during $30 \mathrm{~min}$. The SB grafted films (SB-UV) were then obtained (Fig. 1b).

\subsection{Characterization techniques}

\subsubsection{Water contact angle and surface energies determination}

The water contact angle and surface free energy measurements were performed at room temperature in an OCA 20 contact angle measurement unit from Dataphysics. The contact angle is defined as the angle formed by the baseline and the tangent to the drop contour at the three-phase point. This value is specific for any given system being determined by the interactions of the three interfaces $[18,19]$. Water contact angles $(\theta)$ were assessed by static contact angle measurements using the sessile drop method.

Surface free energy $\left(\gamma_{\mathrm{SV}}\right)$ values as well as the dispersive $\left(\gamma_{\mathrm{s}}^{\mathrm{d}}\right)$ and polar $\left(\gamma_{\mathrm{s}}^{\mathrm{p}}\right)$ components were obtained according to the Owens-Wendt-Rabel and Kaelbe method
(OWRK) [20] by static contact angle measurements with three liquids: water, diiodomethane and formamide. All measurements were performed on the air-facing surfaces of the samples with the three liquids using the sessile drop method. Nine measurements on different points were performed on each sample from which the mean static contact angle and its standard deviation were determined. The surface energies were assessed for all the prepared films.

\subsubsection{Hydrophobic recovery analysis}

Samples were aged in air, wrapped in aluminum foil to minimize hydrocarbon contamination and examined after $1,2,6,14$ and 30 days. The hydrophobicity recovery was evaluated by water contact angle determination, as previously described.

\subsubsection{X-ray photoelectron spectroscopy}

X-ray photoelectron spectroscopy (XPS) measurements were made on a VGS ESCALAB 200A spectrometer with an $\mathrm{Al} \mathrm{K} \alpha \mathrm{X}$-ray source. The operation conditions were set to $15 \mathrm{kV}$. The binding energy scale was fixed by assigning a binding energy of $285.0 \mathrm{eV}$ to the $-\mathrm{CH}_{2}-$ carbon (1s) peak. The samples were analyzed at a take-off angle of $0^{\circ}$ relative to the normal of the surface. The $\mathrm{C} 1 \mathrm{~s}, \mathrm{O} 2 \mathrm{~s}$, and N1s envelopes were analyzed and peak-fitted using a combination of Gaussian and Lorentzian peak shapes obtained from the XPSpeak 4.1 software [21].

\subsubsection{Atomic force microscopy}

The morphology of the surfaces was evaluated by atomic force microscopy (AFM). AFM was performed in a 
Nanoscope IVa Veeco Metrology using the tapping mode (scan size $4.0 \mu \mathrm{m}$, scan rate $1.0 \mathrm{~Hz}$ ). The average roughness $\left(\mathrm{R}_{\mathrm{a}}\right)$ was calculated directly from $700 \times 700 \mathrm{~nm}^{2}$ sized height AFM images at three different positions for each sample.

\subsubsection{Blood compatibility}

Blood compatibility assays were performed in vitro accordingly to the International Standard Organization (ISO) 10993-4 [22]. Both the haemolytic potential and thrombogenicity of the prepared films were evaluated.

2.3.5.1 Haemolytic potential The haemolysis tests were performed as described in American Society for Testing and Materials (ASTM) F 756-00 standard [23]. Samples with a surface area of $21 \mathrm{~cm}^{2}$ were placed in polypropylene test tubes and $7 \mathrm{~mL}$ of PBS $(10 \mathrm{M}, \mathrm{pH}=7.4)$ were added. After $72 \mathrm{~h}$ of incubation at $37{ }^{\circ} \mathrm{C}$, the PBS was removed and the samples were left to dry. After drying, $7 \mathrm{~mL}$ of PBS and $1 \mathrm{~mL}$ of diluted anticoagulated rabbit blood (ACD blood) $(10 \pm 1 \mathrm{mg} / \mathrm{mL})$ was added to each sample. Positive and negative controls were prepared by adding the same amount of ACD blood to $7 \mathrm{~mL}$ of water and PBS, respectively. The tubes were placed at $37{ }^{\circ} \mathrm{C}$ for $3 \mathrm{~h}$ and gently inverted twice every $30 \mathrm{~min}$ to maintain contact between the blood and the material. After incubation, the fluid was transferred to a suitable tube and centrifuged at $700-800 \times g$ for $15 \mathrm{~min}$. The amount of haemoglobin released by haemolysis was assessed by measuring the optical densities of the supernatants at $540 \mathrm{~nm}$, using a spectrophotometer UV-vis (Jasco V550). The percentage of haemolysis (or haemolytic index, HI) was calculated as described in Eq. 1.

$H I(\%)=\frac{O D \text { of test sample }-O D(-) \text { control }}{O D(+) \text { control }-O D(-) \text { control }} \times 100$

According to the ASTM F 765-00 [23] materials can be classified as non-haemolytic when $0>\mathrm{HI}>2$, slightly haemolytic when $2>\mathrm{HI}>5$ and haemolytic when $\mathrm{HI}>5$.

2.3.5.2 Thrombogenicity In vitro thrombogenicity assay was assessed by gravimetric analysis, an adaptation of the method described by Imai and Nose [24-26]. Anticoagulated rabbit blood, prepared by adding $1 \mathrm{~mL}$ of ACD solution to $9 \mathrm{~mL}$ of fresh rabbit blood, was also used for this purpose. Before performing the tests, the membranes were immersed in PBS solution $(\mathrm{pH}$ 7.4) at a constant temperature of $37^{\circ} \mathrm{C}$. After $24 \mathrm{~h}$ of incubation, the PBS was removed and $250 \mu \mathrm{L}$ of ACD blood was carefully
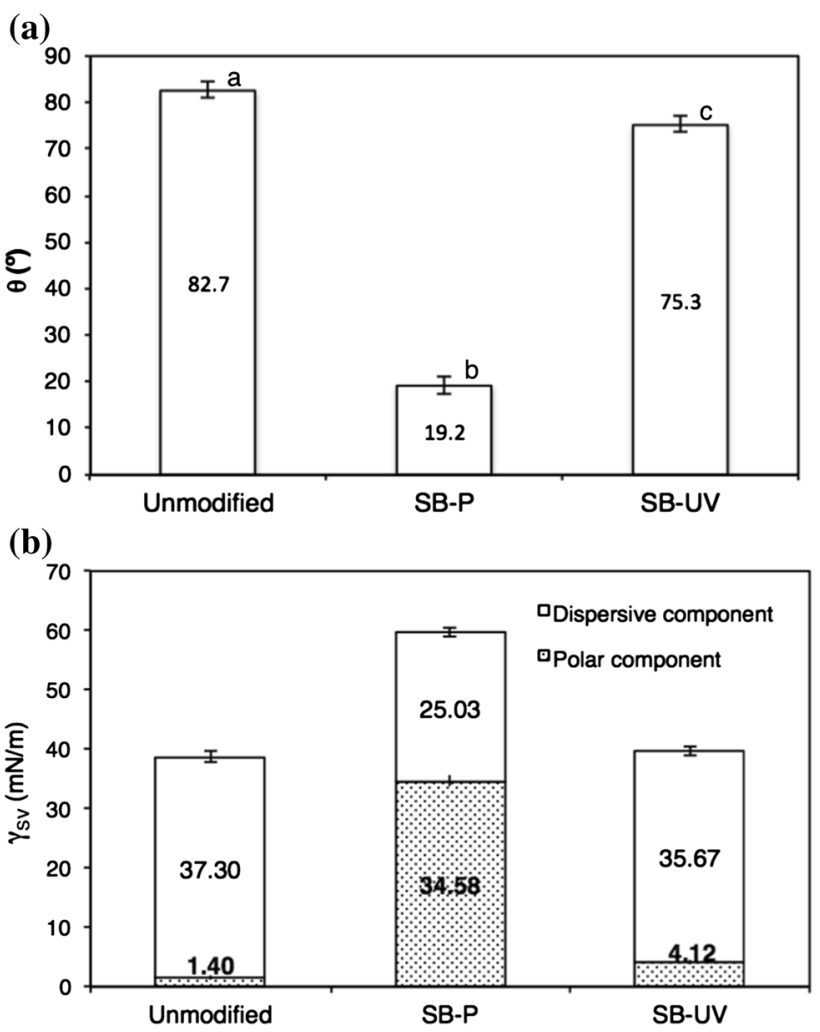

Fig. 2 a Variation of water contact angle of unmodified TPU and grafted TPU either by plasma activation (SB-P) or UV irradiation (SB-UV) (mean $\pm \mathrm{SD}, n=7$ ). Different letters indicate values that are significantly different (one-way ANOVA and Tukey's test, $P<0.05)$. b Surface free energy represented by both dispersive and polar components of unmodified TPU, plasma grafted TPU (SB-P) and UV irradiation grafted TPU (SB-UV)

placed over the surface of the films and also in an empty Petri dish, which acted as a positive control. Blood clotting tests were initiated by adding $25 \mu \mathrm{L}$ of a $0.10 \mathrm{M}$ calcium chloride solution and then stopped after $30 \mathrm{~min}$, by adding $5 \mathrm{~mL}$ of distilled water. The resultant clots were fixed with $1 \mathrm{~mL}$ of a $36 \%$ formaldehyde solution and then dried with tissue paper and finally weighted. The thrombosis degree was calculated and expressed as a percentage in relation to the positive assay.

\subsubsection{Human bone marrow cells adhesion and spreading}

Cell adhesion on the surfaces was evaluated with human bone marrow cells (HBMC). HBMC were harvested using trypsin-EDTA solution and were seeded on the membrane surfaces and incubated at $37{ }^{\circ} \mathrm{C}$ for $24 \mathrm{~h}$. After the incubation period cells were fixed with a paraformaldehyde solution (4\% paraformaldehyde) with $0.2 \%$ Triton X 100 , which makes the cell membrane permeable for the dyes. Then the actin cytoskeleton of the cells was stained with Phalloidin Alexa Fluor 488 (green fluorescent dye) [27]. 
Confocal laser scanning microscopy in the fluorescence mode was used to visualize the cell spreading.

\subsubsection{Statistical analysis}

Statistical analysis was carried out using GraphPad Prism 6 . Results were expressed as the mean \pm standard deviation, compared through one-way analysis of variance (ANOVA, $P=0.05$ ) and two-way analysis of variance (ANOVA, $P=0.05$ ). Tukey's multiple comparisons test was used to identify the significantly different means.

\section{Results and discussion}

\subsection{Water contact angle and surface free energy}

Contact angle measurements were performed for the unmodified and grafted surfaces in order to evaluate the surface modification reaction. Figure $2 \mathrm{a}$ shows the obtained values of water contact angles for all surfaces. Here can be observed that there are significant differences in the surface wettability of the unmodified TPU surface and SB-grafted surfaces.

In Fig. 2a is demonstrated that both grafting methods led to a surface with lower water contact angle. From this decrease of water contact angle is possible to suggest that after grafting, both plasma and UV methods allowed to obtain a more hydrophilic surface. However, the decrease in the water contact angle was more significant for the films treated with plasma suggesting a higher grafting efficiency.

Surface energy is recognized as an important parameter on polymers adhesion, wettability and even biocompatibility [28]. It is known that polar component includes Coulomb interactions between permanent dipoles and interactions between permanent dipoles and induced dipoles; while disperse interactions are caused by time fluctuations in the charge distribution within the molecules [29]. The surface energies obtained for the different surfaces as well as their polar and dispersive components can be seen in Fig. 2b. These results were obtained by using Owens-Wendt-Rabel and Kaelbe method (OWRK) [20]. Figure $2 b$ shows that surface energy increased after both grafting procedures. This increase can be explained by the introduction of the polar functional groups, $\mathrm{SO}_{3}{ }^{-}$groups ascribed to the SB monomer. For the same reason an increase in the polar component of surface energy on the modified surfaces is clearly observed. As expected, and as verified in previous works [30], the increase of surface energy and its polar component is more significant for the plasma activated films, which supports the suggestion that plasma treatment leads to higher grafting yields.

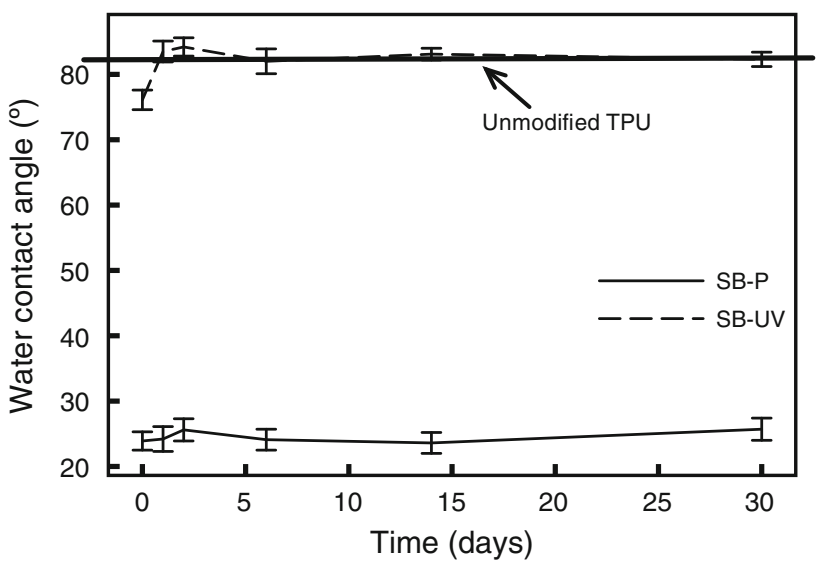

Fig. 3 Values of water contact angles, measured along time, for unmodified TPU membrane, plasma grafted TPU (SB-P) and UV irradiation grafted TPU (SB-UV)

\subsection{Hydrophobic recovery analysis}

Water contact angles were measured along 30 days in order to assess hydrophobic recovery of TPU surfaces. It is known that surfaces that undergo a surface modification procedure by a radiation process partially recover their hydrophobicity along time [6, 30, 31]. Figure 3 illustrates the contact angles variation with time, for the unmodified surface and also for the grafted SB surfaces.

The hydrophobicity recovery study showed that for both modifications (SB-P and SB-UV) the contact angle stabilizes after 6 days. As previously sated, modification by plasma resulted in a more hydrophilic surface (with lower contact angle). Also, this angle value remains constant along time, which suggests that a uniform polymeric layer of SB was coated on the surfaces. The hydrophobicity recovery of a surface might be explained by air contamination or even by surface rearrangements [32-34]. These may decrease the active sites induced to the surface by the plasma or UV surface activation and therefore the water contact angle increases. In this study, we could conclude that such effects were not significant for any of the samples.

\subsection{X-ray photoelectron spectroscopy (XPS)}

XPS spectra of the grafted films (SB-P and SB-UV) as well as of the unmodified TPU films were recorded in order to access the elemental and chemical composition of the films surfaces. Figure 4 illustrates the $\mathrm{C} 1 \mathrm{~s}, \mathrm{~N} 1 \mathrm{~s}, \mathrm{O} 1 \mathrm{~s}$ and $\mathrm{S} 2 \mathrm{p}$ spectra obtained and shows their relative composition ratio based on the area of the peaks.

The C1s peak, as shown in Fig. 4a, could be resolved in three components: the hydrocarbon $(\mathrm{C}-\mathrm{C}$ and $\mathrm{C}-\mathrm{H})$ peak at 

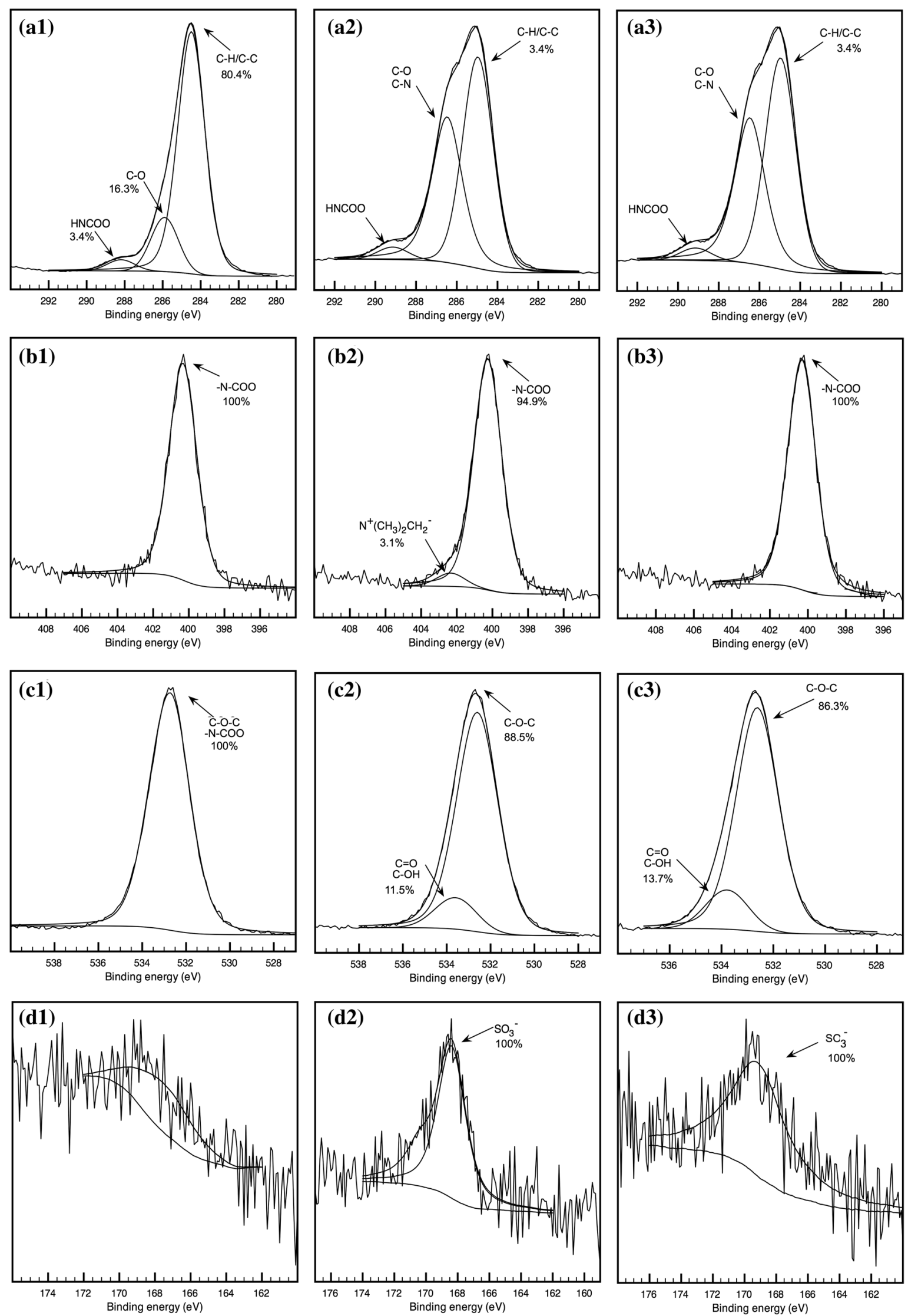
4Fig. 4 XPS spectra of C1s (a) N1s (b) O1s (c) and S2p (d) for the unmodified TPU (1) and for the SB grafted surfaces by plasma activation (2) and by UV irradiation (3). Peak area (\%) based on the area of each peak for the unmodified and both SB grafted TPU are also shown

$284.5 \mathrm{eV}$, the ether peak $(\mathrm{C}-\mathrm{O}-\mathrm{C})$ at $285.9 \mathrm{eV}$ and the urethane peak $(\mathrm{NH}-\mathrm{COO})$ at $288.0 \mathrm{eV}$. It can be seen that both SB grafted TPU, Fig. $4 \mathrm{a} 2$ and a3), has a higher peak area around $286.5 \mathrm{eV}$ due to the introduction of the $\mathrm{C}-\mathrm{N}$ groups from the $\mathrm{SB}$ monomer.

The N1s peak of the unmodified TPU (Fig. 4b1) is resolved by one single peak ascribed to the urethane peak (NCOO) [6, 35]. After grafting, for SB-P two peaks can be identified: the urethane peak (NH-COO) at $400 \mathrm{eV}$ [35] and the SB ammonium nitrogen peak $\left(\mathrm{N}^{+}\left(\mathrm{CH}_{3}\right)_{2} \mathrm{CH}_{2}{ }^{-}\right)$at around $402 \mathrm{eV}$ [17]. However, SB-UV N1s peak shows only one peak slightly wider than the unmodified TPU, meaning that this grafting method is less efficient than the plasma method.

The O1s peak (Fig. 4c) of both SB grafted TPU consists of two peaks, while the unmodified TPU consists of only one O1s peak. Furthermore, both SB grafted TPU have a wider binding energy of O1s peak than unmodified TPU. This can be due to the combination of the urethane peak $(\mathrm{NH}-\mathrm{COO})$ and the ether peak $(\mathrm{C}-\mathrm{O}-\mathrm{C})$ at around $532 \mathrm{eV}$. For both SB grafted surfaces one more peak is visible at $533.60 \mathrm{eV}$ due to the contribution of the oxygen from the sulfobetaine monomer $\left(\mathrm{SO}_{3}{ }^{-}\right)$. This second $\mathrm{C}-\mathrm{O}$ peak is higher for SB-P relatively to SB-UV, which confirms the higher grafting efficiency of the plasma procedure.

Finally, looking at Fig. 4d, the S2p spectra, a new peak shows at a binding energy of $168.4 \mathrm{eV}$ when compared to the unmodified films. This peak is also attributed to the sulfobetaine monomer $\left(\mathrm{SO}_{3}{ }^{-}\right)$. Once again, the $\mathrm{SB}$ peak obtained for SB-P is more evident than the SB-UV peak.

From the presented results it can be clearly confirmed the assumptions made from the surface energy results. Summarily, the grafting of SB onto the TPU surface was achieved, particularly when plasma is used, meaning that the SB grafting is more efficient by using the plasma activation method.

\subsection{Atomic force microscopy}

AFM analysis was used to assess surface topography of the grafted and unmodified films. The 3-dimensional (3-D) AFM images of the TPU films are presented in Fig. 5. The 3D images show that after surface grafting, smoother surfaces are obtained.

Nevertheless, the difference in surface topography is more notorious when plasma surface activation is used to graft SB. In order to better quantify these differences in surface topography, surface roughness $\left(R_{a}\right)$ was determined. $R_{a}$ was obtained directly from the AFM images and is displayed in Fig. 5.

As expected, SB-P and SB-UV surfaces show lower $\mathrm{R}_{\mathrm{a}}$ values due the presence of the SB layer and according to oneway ANOVA analysis, the value of $R_{a}$ of the unmodified TPU surface is significantly different from SB-grafted surfaces. Comparing the two SB grafting methods, statistical analysis shows that there are no significant differences between the grafted surfaces, although the obtained results suggest that SB grafting by plasma activation is more efficient since higher densities of grafted monomer result in smoother surfaces [33]. This modification on roughness values also allows to confirm the occurrence of the surface modification and is coherent with the results obtained in XPS analysis and contact angle measurements. In a previous work [30] the same difference between both grafting methods (UV and plasma) has observed for the grafting of other monomers onto the same TPU base material.

\subsection{Haemolysis}

The haemolysis results obtained for the unmodified and grafted TPU surfaces are shown in Fig. 6. According to the
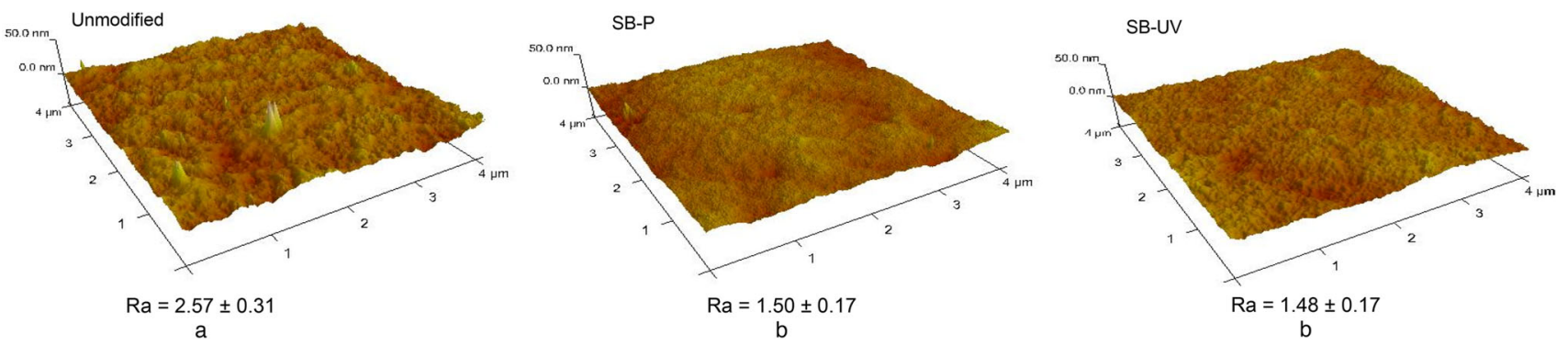

Fig. 5 Atomic force micrographs of the unmodified TPU and the SB-grafted surfaces (mean $\pm \mathrm{SD}, n=6$ ). Different letters indicate values that are significantly different (one-way ANOVA and Tukey's test, $P<0.05$ ) 


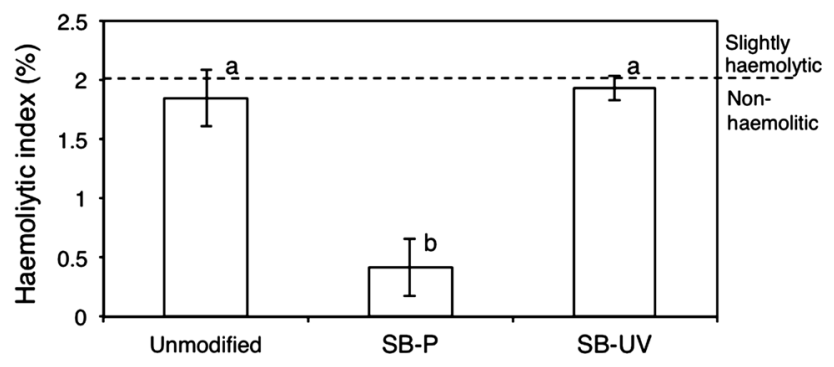

Fig. 6 Haemolytic index of the unmodified and the SB-grafted surfaces (mean $\pm \mathrm{SD}, n=3$ ). Different letters indicate values that are significantly different (one-way ANOVA and Tukey's test, $P<0.05)$

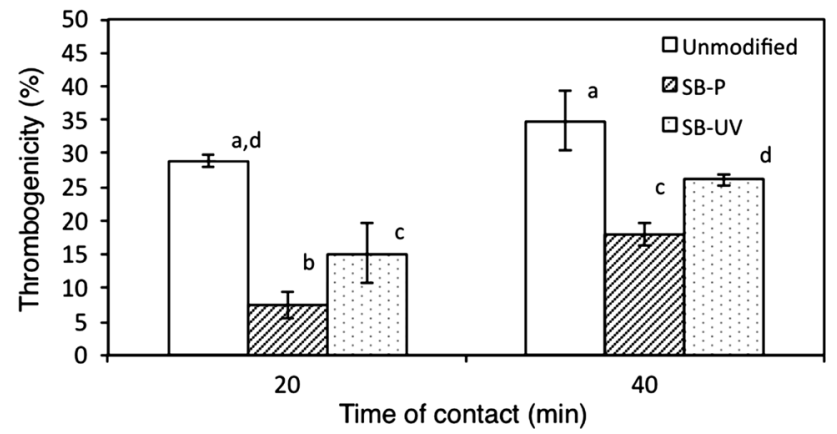

Fig. 7 Thrombogenicity for the unmodified TPU and each surface grafting (mean $\pm \mathrm{SD}, n=3$ ). Different letters indicate values that are significantly different (two-way ANOVA and Tukey's test, $P<0.05)$

ASTM F 756-00 [23] all samples were found to have no haemolytic effect (haemolytic index lower than $2 \%$ ).

The haemolytic index (HI) of the unmodified TPU is not significantly different from SB-UV surface, which indicates that UV treatment does not significantly influences the HI. On the other hand, the HI value for the SB-P is significantly lower than the unmodified and SB-UV TPU, suggesting that plasma treatment of the original material improves its haemocompatibility. This can be explained by the higher hydrophobicity of unmodified and SB-UV TPU surfaces, which might support protein adhesion, weakening the membrane of the erythrocytes, promoting lysis and haemoglobin release [36].

According to these results, all samples were found to be suitable to use in biomedical applications, with special emphasis on the SB-P films.

\subsection{Thrombosis}

The thrombogenicity results are gathered in Fig. 7. These results show that for blood contact times of 20 and $40 \mathrm{~min}$, the grafted surfaces present a lower thrombogenicity when compared to the unmodified films. For 20 min of contact time with blood, the thrombogenicity for all the TPU surfaces (unmodified TPU and SB-UV and SB-P grafted surfaces) was below $30 \%$ and statistical analysis shows significant differences. For $40 \mathrm{~min}$ of contact time, the thrombogenicity slightly increases and statistical analysis also shows significant differences between all assays. Therefore, the obtained results suggest that the SB layer on the TPU's surfaces slightly suppresses the clotting process.

Previous studies reported that hydrophilic surfaces tend to reduce protein adsorption, a process that is related with thrombus formation. As previously stated, the measurement of water contact angles indicated an increase of the surface hydrophilicity with grafting and, consequently, these modified surfaces are more efficient repelling protein adsorption and avoiding thrombus formation. This reduction on the thrombosis values is also supported by the decrease in roughness shown by the AFM analysis, since rough surfaces tend to increase protein adsorption [37, 38].

Comparing the two SB grafting methods, SB-P sample showed lower thrombogenicity values justified by its
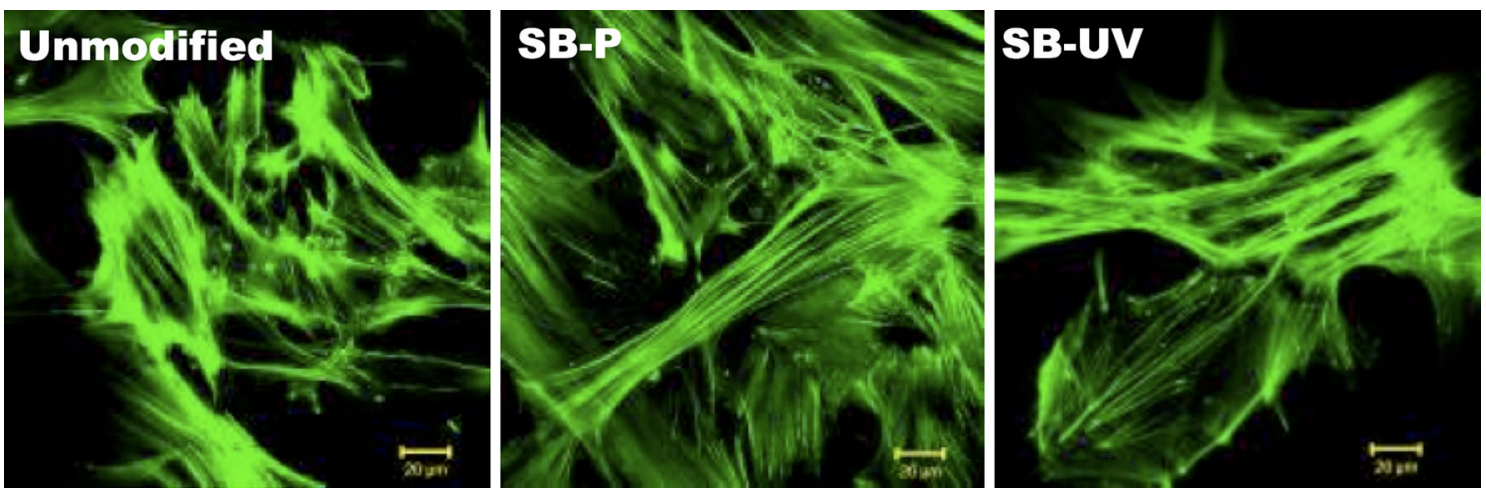

Fig. 8 HBMC cultured on the unmodified and SB-grafted TPUs membranes surfaces 
higher hydrophilicity and lower surface roughness. Furthermore, these results lead to the conclusion that modified TPU surfaces perform better than the unmodified surface, and all materials are suitable to be in contact with blood.

\subsection{Human bone marrow cells adhesion and spreading}

Cell adhesion on the surfaces was evaluated with human bone marrow cells (HBMC). Cells were fixed after a culture period of 1 day and the actin cytoskeleton was stained with Phalloidin Alexa Fluor 488 conjugate (fluorescent dye). Cell adherence and spreading on the grafted and unmodified surfaces showed similar patterns, as can be seen in Fig. 8.

Cells showed a good adhesion and developed a wellestablished actin cytoskeleton on the unmodified TPU (control surface), as already reported in previous studies involving 3T3 mouse fibroblasts [39]. On SB-UV grafted TPU, cells showed a good cell adherence. On SB-P, cells also developed a good cell adhesion with a well-established cytoskeleton. In the later surface the cells look more spread and flatten then on SB-UV surface. This slight difference in the cell spreading might be associated with the density of the grafting. The surface density of sulfonic groups in a surface is known to be responsible for cells flattening. The higher the density of sulfonic groups, the higher the tendency of cells to flatten on the substrate surface [40].

\section{Conclusions}

Surface modification of Elastollan ${ }^{\circledR} 1180$ A50 films with SB was successfully achieved via two methods: argon plasma surface activation and UV grafting. Surface grafting created significantly smoother surfaces, as was proved by AFM analysis. XPS proved the SB grafting by the presence of $\mathrm{SO}_{3}{ }^{-}$and the ammonium nitrogen peaks $\left(\mathrm{N}^{+}\left(\mathrm{CH}_{3}\right)_{2-}\right.$ $\mathrm{CH}_{2}{ }^{-}$). Also, grafted films exhibited an improvement in hydrophilicity due to the introduction of SB polar groups and lower roughness, especially when plasma surface activation was used which indicates a higher density of grafting for SB-P, which was upheld by HBMC adhesion tests. Haemocompatibility results revealed good blood compatibility for all samples. Both thrombosis and haemolysis tests proved that the films were not responsible for any damage in blood, mainly for the grafted surfaces. This means that grafting of SB on the surfaces improves the blood-compatibility profile of the original TPU surface.

Acknowledgments The present study was supported by the European Commission through the specific targeted research project CellForce of the 6th FP (Contract No.: NMP4-CT-2005-016626).

\section{References}

1. Horbett TJ, Brash JL. Proteins at interfaces: an overview. In: Horbett TJ, Brash JL, editors. Proteins at interfaces II: fundamentals and applications. Washington, DC: American Chemical Society; 1995. p. 1-25.

2. Alves P, Coelho JFJ, Haack J, Rota A, Bruinink A, Gil MH. Surface modification and characterization of thermoplastic polyurethane. Eur Polym J. 2009;45:1412-9.

3. Hylton DM, Shalaby SW, Latour RA. Direct correlation between adsorption induced changes in protein structure and platelet adhesion. J Biomed Mater Res. 2005;73A:349-58.

4. He D, Susanto H, Ulbricht M. Photo-irradiation for preparation, modification and stimulation of polymeric membranes. Prog Polym Sci. 2009;34:62-98.

5. Siow KS, Britcher L, Kumar S, Griesser HJ. Plasma methods for the generation of chemically reactive surfaces for biomolecule immobilization and cell colonization-a review. Plasma Process Polym. 2006;3:392-418.

6. Alves P, Sousa HC, Gil MH. Surface grafting polymerization of thermoplastic polyurethane by low pressure plasma treatment to improve hydrophilicity. J Appl Polym Sci. 2011;122:2302-8.

7. Inagaki N. Plasma surface modification and plasma polymerization. Basel: Techomic publishing co Inc.; 1996. p. 1-19.

8. Hu S, Ren X, Li MBCSG, Allbritton N. Surface-directed, graft polymerization within microfluidic channels. Anal Chem. 2004;76:1865-70.

9. Zheng Y, Gurgel PV, Carbonell RG. Effects of UV exposure and initiator concentration on the spatial variation of poly(glycidyl methacrylate) grafts on nonwoven fabrics. Ind Eng Chem Res. 2011;50:6115-23.

10. Deng J, Wang L, Liu L, Yang W. Developments and new applications of UV-induced surface graft polymerizations. Prog Polym Sci. 2009;34:156-93.

11. Ebara M, Hoffman J, Stayton P, Hoffman A. Surface modification of microfluidic channels by UV-mediated graft polymerization of non-fouling and 'smart' polymers. Radiat Phys Chem. 2007;76:1409-13.

12. Wong I, Ho C-M. Surface molecular property modifications for poly(dimethylsiloxane) (PDMS) based microfluidic devices. Microfluid Nanofluid. 2009;7:291-306.

13. Geiger B, Spatz JP, Bershadsky AD. Environmental sensing through focal adhesions. Nat Rev Mol Cell Biol. 2009;10:21-33.

14. Kumar S, Maxwell IZ, Heisterkamp A, Polte TR, Lele TP, Salanga M, Mazur E, Ingber DE. Viscoelastic retraction of single living stress fibers and its impact on cell shape, cytoskeletal organization, and extracellular matrix mechanics. Biophys J. 2006;90:3762-73.

15. Kaiser J-P, Bruinink A. Investigating cell-material interactions by monitoring and analysing cell migration. J Mater Sci Mater Med. 2004;15:429-35.

16. Tymchenko N, Wallentin J, Petronis S, Bjursten LM, Kasemo B, Gold J. A novel cell force sensor for quantification of traction during cell spreading and contact guidance. Biophys J. 2007;93:335-45.

17. Yuan J, Huang X, Li P, Li L, Shen J. Surface-initiated RAFT polymerization of sulfobetaine from cellulose membranes to improve hemocompatibility and antibiofouling property. Polym Chem. 2013;4:5074-85.

18. Correia NT, Ramos JJM, Saramago BJV, Calado JCG. Estimation of the surface tension of a solid: application to a liquid crystalline polymer. J Colloid Interface Sci. 1997;189:361-9.

19. Chibowski E. Surface free energy of a solid from contact angle hysteresis. Adv Colloid Interface Sci. 2003;103:149-72. 
20. Owens D, Wendt R. Estimation of the surface free energy of polymer. J Appl Polym Sci. 1969;13:1711-7.

21. Raymund Kwok WM. XPSPEAK, XPS Peak Fitting Program for WIN95/98, Version 4.1. Hong Kong: Department of Chemistry, The Chinese University of Hong Kong; 2000.

22. ISO. 10993-4: Biological evaluation of medical devices, part 4: selection of tests for interaction with blood. Geneva, Switzerland: International Organization for Standardization; 2002.

23. ASTM F 756-00. Standard practice for assessment of hemolytic properties of materials. Philadelphia: American Society for Testing and Materials; 2000.

24. Imai Y, Nose Y. A new method for evalution of antithrombogenicity of materials. J Biomed Mater Res. 1972;6:165-72.

25. Ferreira P, Pereira R, Coelho JFJ, Silva AFM, Gil MH. Modification of the biopolymer castor oil with free isocyanate groups to be applied as bioadhesive. Int J Biol Macromol. 2007;40:144-52.

26. Besteiro MC, Guiomar AJ, Gonçalves CA, Bairos VA, Pinho $\mathrm{MN}$, Gil MH. Characterization and in vitro hemocompatibility of bi-soft segment, polycaprolactone-based poly(ester urethane urea) membranes. J Biomed Mater Res A. 2010;93A:954-64.

27. Kaiser J-P, Wick P, Manser P, Spohn P, Bruinink A. Single walled carbon nanotubes (SWCNT) affect cell physiology and cell architecture. J Mater Sci Mater Med. 2008;19:1523-7.

28. Kwok SCH, Wang J, Chu PK. Surface energy, wettability, and blood compatibility phosphorus doped diamond-like carbon films. Diam Relat Mater. 2005;14:78-85.

29. Interfacial chemistry, operating manual dataphysics OCA. DataPhysics Instruments GmbH, Germany; 2002. p. 1-4

30. Alves P, Pinto S, Kaiser J-P, Bruinink A, Sousa HC, Gil MH. Surface grafting of a thermoplastic polyurethane with methacrylic acid by previous plasma surface activation and by ultraviolet irradiation to reduce cell adhesion. Colloids Surfaces B. 2011;82:371-7.
31. Pinto S, Alves P, Matos CM, Santos AC, Rodrigues LR, Teixeira JA, Gil MH. Poly(dimethyl siloxane) surface modification by low pressure plasma to improve its characteristics towards biomedical applications. Colloids Surfaces B. 2010;81:20-6.

32. Wang P, Tan K, Ho C, Khew M, Kang E. Surface modification of natural rubber latex films by graft copolymerization. Eur Polym J. 2000;36:1323-31.

33. Qiu Y, Klee D, Plüster W, Severich B, Höcker H. Surface modification of polyurethane by plasma-induced graft polymerization of poly(ethylene glycol) methacrylate. J Appl Polym Sci. 1996;62:2373-82.

34. Lappan U, Buchhammer H-M, Lunkwitz K. Surface modification of poly(tetrafluoroethylene) by plasma pretreatment and adsorption of polyelectrolytes. Polymer. 1999;40:4087-91.

35. Shen W, Li Z, Liu Y. Surface chemical functional groups modification of porous carbon. Recent Patents Chem Eng. 2008;1:27-40.

36. Masters KS. The haemocompatibility of polyurethane-hyaluronic acid copolymers. Biomaterials. 2007;29:150-60.

37. Chen H, Zhang Z, Chen Y, Brook MA, Sheardown H. Protein repellent silicone surfaces by covalent immobilization of poly(ethylene oxide). Biomaterials. 2005;26:2391-9.

38. Zhao C, Liu X, Nomizu M, Nishi N. Blood compatibility aspects of DNA-modified polysulfone membrane-protein adsorption and platelet adhesion. Biomaterials. 2003;24:3747-55.

39. Alves P, Kaiser J-P, Haack J, Salk N, Bruinink A, Sousa HC, Gil MH. Surface modification of thermoplastic polyurethane in order to enhance reactivity and avoid cell adhesion. Colloid Polym Sci. 2009;287:1469-74.

40. Kowalczyńska HM, Nowak-Wyrzykowska M, Inkielman M, Stołowska L, Marciniak E. The shape of cells adhering to sulfonated copolymer surfaces. Cell Mol Biol Lett. 2005;10:1-9. 